\title{
Acceleration of Calculation of Nuclear Heating Distributions in ITER Toroidal Field Coils Using Hybrid Monte Carlo/Deterministic Techniques
}

\author{
Ahmad M. Ibrahim ${ }^{\mathrm{a}}$, Eduard Polunovskiy ${ }^{\mathrm{b}}$, Michael J. Loughlin ${ }^{\mathrm{b}}$, Robert E. Grove ${ }^{\mathrm{a}}$, \\ Mohamed E. Sawan ${ }^{\mathrm{c}}$ \\ ${ }^{a}$ Oak Ridge National Laboratory, P.O. Box 2008, Oak Ridge, TN 37831, USA \\ ${ }^{b}$ ITER Organization, Route de Vinon Sur Verdon, 13067, St. Paul Lez Durance, France \\ ${ }^{c}$ University of Wisconsin-Madison, 1500 Engineering Dr., Madison, WI, 53706, USA
}

\begin{abstract}
Because the superconductivity of the ITER toroidal field coils (TFC) must be protected against local overheating, detailed spatial distribution of the TFC nuclear heating is needed to assess the acceptability of the designs of the blanket, vacuum vessel (VV), and VV thermal shield. Accurate Monte Carlo calculations of the distributions of the TFC nuclear heating are challenged by the small volumes of the tally segmentations and by the thick layers of shielding provided by the blanket and VV. To speed up the MCNP calculation of the nuclear heating distribution in different segments of the coil casing, ground insulation, and winding packs of the ITER TFC, the ITER Organization (IO) used the MCNP weight window generator (WWG). The maximum relative uncertainty of the tallies in this calculation was $82.7 \%$. In this work, this MCNP calculation was repeated using variance reduction parameters generated by the Oak Ridge National Laboratory AutomateD VAriaNce reducTion Generator (ADVANTG) code and both MCNP calculations were compared in terms of computational efficiency and reliability. Even though the ADVANTG MCNP calculation used less than one-sixth of the computational resources of the IO calculation, the relative uncertainties of all the tallies in the ADVANTG MCNP calculation were less than $6.1 \%$. The nuclear heating results of the two calculations were significantly different by factors between 1.5 and 2.3 in some of the segments of the furthest winding pack turn from the plasma neutron source. Even though the nuclear heating in this turn may not affect the ITER design because it is much smaller than the nuclear heating in the turns that are closer to the plasma source, it is our recommendation to adopt the utilization of ADVANTG in similar calculations to increase the reliability of MCNP calculations of detailed distributions of nuclear responses.
\end{abstract}

Keywords: ITER shielding, toroidal field coils, nuclear heating distribution, hybrid Monte Carlo/deterministic techniques.

\section{Introduction}

The ITER tokamak design uses 18 toroidal field coils (TFC) as part of the magnetic confinement system for the plasma. The TFC must be cooled to cryogenic temperatures during ITER operation to avoid the transition of the winding pack superconducting material $\left(\mathrm{Nb}_{3}\right.$ Sn-type) to the normal resistive state. The TFC are cooled with supercritical helium in the temperature range of 4.2 to $4.5 \mathrm{~K}$. The permanent heat load is caused by the heating effect of nuclear radiation in both the winding packs and the coil casings [1]. The design decision was made during the ITER Engineering Design Activities phase to limit the nuclear heating from the reference 500 MW plasma to levels comparable with that of the alternating current losses, in the range of 10 to $20 \mathrm{~kW}$ when averaged over an $1800 \mathrm{~s}$ plasma cycle [1]. However, this global integrated nuclear heating limit contains implicit assumptions about the spatial distribution of the nuclear heat. To avoid localized losses of superconductivity, the TFC must be protected against local overheating. Therefore, it is necessary to determine the spatial distribution of the nuclear heating among the coil case components and the winding pack turns in order to properly interpret the total integrated value and the design margins [1].
The poloidal and radial distributions of nuclear heating in the TFC were calculated ITER Organization (IO) using an updated version of the ITER Clite model, which is a simplified global model for a $40^{\circ}$ regular sector of the ITER machine [2]. This calculation used the MCNP [3] weight window generator (WWG) [4] because it is difficult to achieve acceptable statistical uncertainties in such detailed calculations without the use of strong methods of variance reduction. The calculation of the TFC nuclear heating detailed distributions is very challenging because of the small volumes of the tally segments inside the different components of the TFC and the thick layers of shielding provided by the blanket and the vacuum vessel (VV). In this work, the calculation of the ITER TFC nuclear heating spatial distribution was repeated using the Oak Ridge National Laboratory (ORNL) AutomateD VAriaNce reducTion Generator (ADVANTG) code, which automates the generation of variance reduction parameters for MCNP5 simulations [5]. The tally values and the statistical uncertainties of the ADVANTG MCNP calculation were compared with the corresponding results of the MCNP calculation that used the MCNP WWG. While this work compares the computational time and computational efficiency of the

ibrahimam@ornl.gov 
two approaches, our primary focus is on assessing the reliability of the two approaches for being conveniently and regularly used in such important analyses. It is worth mentioning that the interpretation of the nuclear heating results and how these results affect the ITER design are outside the scope of this work.

\section{Toroidal field coils tally segments}

In the Clite model, the TFC was divided into 32 equal length poloidal segments, as shown in Fig. 1.

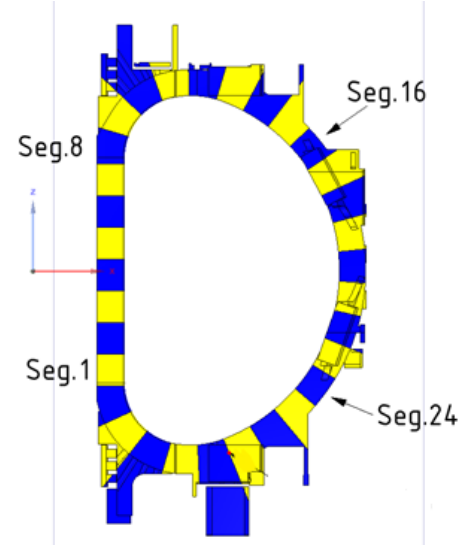

Fig. 1. Poloidal segmentation of a TFC.

The MCNP calculation involved 544 tallies because the nuclear heating was calculated in each of the three components (front, side, and back) of the coil casing and the ground insulation and in each of the 11 coil turns of the winding packs.

\section{Methodology}

ADVANTG automatically generates space- and energy-dependent mesh-based variance reduction parameters for MCNP5. ADVANTG uses threedimensional discrete ordinates calculations performed by the Denovo package [6] to implement either the Consistent Adjoint Driven Importance Sampling (CADIS) hybrid Monte Carlo (MC)/Deterministic method [7] or the Forward-Weighted CADIS (FWCADIS) method [8]. Based on the results of the deterministic calculation(s), the CADIS and FW-CADIS methods provide a prescription for generating space- and energy-dependent weight-window targets and a consistent biased source distribution. The CADIS method was developed for accelerating individual tallies, whereas FW-CADIS can be applied to multiple tallies and mesh tallies.

The FW-CADIS method was used to improve the $\mathrm{MC}$ estimate of the nuclear heating in all the tallies of this analysis. FW-CADIS uses the inverse of the responses calculated from a forward deterministic calculation to weight the adjoint source strengths in the adjoint deterministic calculation. The adjoint fluxes are then used to calculate the source biasing and weightwindow parameters for the $\mathrm{MC}$ calculation.

To implement either the CADIS or FW-CADIS method, an adjoint source is defined to represent the response of the tallies being calculated through the MC simulation. For calculating the spatial distribution of the nuclear heating in the TFC, the adjoint source was defined as the sum of the neutron and photon heating in all the TFC components including the coil casing, insulators, and the winding packs cells. The Denovo forward and adjoint calculations used 46-neutron/21gamma FENDL-2.1 library and a Cartesian mesh. The mesh had 102 bins in the $x$ direction, 105 bins in the $y$ direction, and 119 bins in the $z$ direction. The ADVANTG unfolding option was used to expand the $40^{\circ}$ ITER model to a full $360^{\circ}$ model for the forward and adjoint calculations. The mesh elements had variable sizes. Elements that surrounded the plasma source and the two TFC in the original $40^{\circ}$ sector had side lengths of approximately $15 \mathrm{~cm}$. Away from this region, the side lengths were approximately $70 \mathrm{~cm}$. Each of the two Denovo calculations used approximately 1.2 CPU-days.

The results of the MCNP calculation that used the weight windows generated by MCNP WWG were provided by the ITER Organization [2]. For quality assurance the results were also calculated without using either the MCNP WWG or ADVANTG. Even though this calculation used the MCNP implicit capture options, which is turned on by default in MCNP calculations, it will be expressed as the analog calculation in this paper for convenience.

\section{Results}

\subsection{Computational Time and Efficiency}

The MCNP calculation that used variance reduction parameters generated by ADVANTG only used 25 CPUdays on a Unix computational cluster. The ITER Organization (IO) used approximately 163 CPU-days for the MCNP calculation that utilized the weight windows generated by the MCNP WWG. The analog MCNP calculation used approximately 195 CPU-days. Table 1 shows the minimum, average, and maximum of the relative uncertainties $(R)$ of the 544 tallies in each calculation.

Table 1. Relative uncertainties $(R)$ of detailed spatial distribution of TFC nuclear heating. All values are at the $1 \sigma$ level.

\begin{tabular}{lccc}
\hline & Analog & $\begin{array}{c}\text { MCNP } \\
\text { WWG }\end{array}$ & ADVANTG \\
\hline Min. $R$ & $0.7 \%$ & $0.3 \%$ & $0.2 \%$ \\
Average $R$ & $15.8 \%$ & $5.6 \%$ & $1.4 \%$ \\
Max. $R$ & $81.3 \%$ & $82.7 \%$ & $6.1 \%$ \\
\hline
\end{tabular}


All the relative uncertainties of the ADVANTG case are $<10 \%$, which is usually considered acceptable for MCNP calculations. Conversely, the fraction of the tallies that had relative uncertainties $>10 \%$ was $13 \%$ with the MCNP WWG and 57\% with analog MCNP. Both the analog and MCNP WWG calculations had large variations (two orders of magnitude) in the relative uncertainties. This large variation of relative statistical uncertainties is inconvenient for $\mathrm{MC}$ practitioners because of the difficulty of calculating the computational time necessary for obtaining reliable $\mathrm{MC}$ results. It is not always guaranteed that all the relative uncertainties will decrease monotonically with increasing computational time if the MCNP calculations are not using optimum variance reduction parameters. Moreover, when strong variance reduction parameters such as the weight windows of the MCNP WWG are used, the difficulty of convergence of some tallies may be caused by an undersampling in the weight windows of some important regions in space and/or energy. This undersampling can cause the MC calculation to be unreliable [9]. With the analog case, it is expected that the relative uncertainties will monotonically decrease with increasing the computational time. However, it may be unaffordable to use analog MC for this calculation because it would require approximately 64 times longer computational time than the already used 200 CPU-days for all the relative uncertainties to be small enough $(<10 \%)$ to consider it a reliable MC calculation. It is worth mentioning that the calculation of nuclear heating in the TFC was repeated several times with different variations in the geometry and material definitions to optimize the design of the blanket, $\mathrm{VV}, \mathrm{TFC}$, and cryogenic systems in ITER [1, 2].

For a single tally, a well-defined figure of merit (FOM) for the MC calculations is established as

$$
F O M=\frac{1}{R^{2} \cdot T}
$$

where $R$ is the relative uncertainty of the tally and $T$ is the computational time of the MC calculation.

Extending the concept of the FOM of a single tally to several tallies is not straightforward since $R$ is not well defined for a tally distribution. Several efficiency metrics have been suggested in the literature for global MC problems, but a unified MC FOM for several tallies has not yet been established. One concept for mesh tallies with thousands or millions of elements is to define an FOM based on the average $R^{2}$ [9]. With such large numbers of mesh tally elements, MC calculations are often stopped before the solution converges for all the elements. This can be represented as

$$
F O M_{\text {average }}=\frac{1}{\overline{R^{2}} \cdot T}
$$

where $\overline{R^{2}}$ is the mean of the distribution function of the relative variances $\left(R^{2}\right)$ of the tally values.

With smaller numbers of tallies at specific locations, the FOM can be defined as

$$
F O M_{\text {max }}=\frac{1}{R_{\text {max }}{ }^{2} \cdot T}
$$

where $R_{\max }$ is the maximum $R$ in all the tallies. Since this FOM is based on the tally with the slowest convergence rate, it can be used for characterizing the MC simulation efficiency if the user is interested in acquiring low $R \mathrm{~s}$ everywhere. Because convergence of the tallies is required for all the components of the TFC, this FOM based on the maximum relative uncertainty is considered more appropriate for this calculation [10].

Table 2 shows the speedup in the MC calculations provided by the use of either the MCNP WWG or ADVANTG. This speedup is calculated by dividing the FOM calculated using either the average or the maximum relative uncertainty of all the tallies for either the MCNP WWG case or the ADVANTG case by the corresponding FOM of the analog case.

Table 2. Speedups of TFC nuclear heating calculations.

\begin{tabular}{lccc}
\hline & Analog & $\begin{array}{l}\text { MCNP } \\
\text { WWG }\end{array}$ & ADVANTG \\
\hline $\begin{array}{l}\text { Speedup } \\
(\text { average } R \text { ) }\end{array}$ & 1.00 & 9.68 & 929 \\
$\begin{array}{l}\text { Speedup } \\
(\text { maximum } R \text { ) }\end{array}$ & 1.00 & 1.16 & 1395 \\
\hline
\end{tabular}

With an FOM based on the average relative uncertainty, the MCNP WWG provided a speed up factor of approximately 10 for the MC calculation compared to analog MC. However, it did not provide any considerable speedup compared to analog $\mathrm{MC}$ when the more appropriate FOM based on the maximum relative uncertainty was used to assess the efficiency of the MC calculation. Compared with the MCNP WWG case, the ADVANTG calculation provided a factor of approximately 96 speedup based on average relative uncertainty and a factor of approximately 1,200 speedup based on the maximum relative uncertainty. It is necessary to mention that the different MCNP calculations that used the MCNP WWG and ADVANTG for VR were performed on different computing systems at different institutions. However, the computational efficiency comparisons are still very valuable because the factor of 1,200 exceeds any differences that may exist because of the differences in the computational powers of the computing systems.

\subsection{Results in some individual TFC components}

Figure 2 shows the poloidal distribution of the sum of the nuclear heating in all the components of the coil casing and the ground insulation and the sum of the nuclear heating in all the winding pack turns. The relative statistical uncertainties in Fig. 2 were calculated by propagating the uncertainties of the MCNP tallies representing the three components of the coil casing and 
ground insulations and the 11 winding packs turns. These uncertainties were calculated by propagating the uncertainty of individual tallies without considering the covariance between these tallies. Because the same particle history that contributes to one tally can still contribute to another tally, the tallies that were calculated by straight forward uncertainty propagation are not statistically independent. However, the level of underestimation in these uncertainties should be the similar for both the MCNP WWG and the ADVANTG results, so the comparison between the tallies uncertainties is still meaningful. Figure 3 shows the ratio of the ITER IO results that used the MCNP WWG to the ORNL results that used ADVANTG for the same quantities.

For the total heating in the coil casing and the ground insulation, MCNP WWG and ADVANTG results agreed within $3 \sigma$ for all the 32 segments except for the 11 th segment, where the $3.6 \%$ difference was 4.9 times larger than the uncertainty in the average value. For all the segments, both MCNP WWG and ADVANTG results agreed within $3 \sigma$ with the analog results. The analog relative statistical uncertainties varied between $0.7 \%$ and $6.9 \%$. The relative statistical uncertainties of the MCNP WWG case varied between $0.4 \%$ and $21.8 \%$. The relative statistical uncertainties of the ADVANTG case varied between $0.3 \%$ and $2.2 \%$. As shown in Fig. 2, the relative uncertainties of the total nuclear heating in the coil casing and ground insulation were not continuously small for the MCNP calculation that used the MCNP WWG. For the MCNP WWG case, the $21.8 \%$ relative statistical uncertainty of the $20^{\text {th }}$ segment was a factor of 10.1 higher than the corresponding value of the ADVANTG MCNP case. It was even higher than the corresponding uncertainty of the analog case, for which the $4.7 \%$ relative statistical uncertainty was acceptable. It is worth mentioning that the use of strong variance reduction methods is not as urgently needed for this specific tally that includes the front casing of the TFC segment as it is needed for the other TFC components that are more heavily shielded from the plasma source. For this specific tally, the difference between the total nuclear heating calculated in the analog case and the corresponding value of the MCNP WWG case was $24.7 \%$ while the difference between the analog and the ADVANTG cases was only $6.4 \%$. These differences can be explained by the statistical uncertainties, but the high relative uncertainty of this tally with the MCNP WWG case may cause inconvenience in adjusting the time necessary for obtaining reliable results in this analysis.

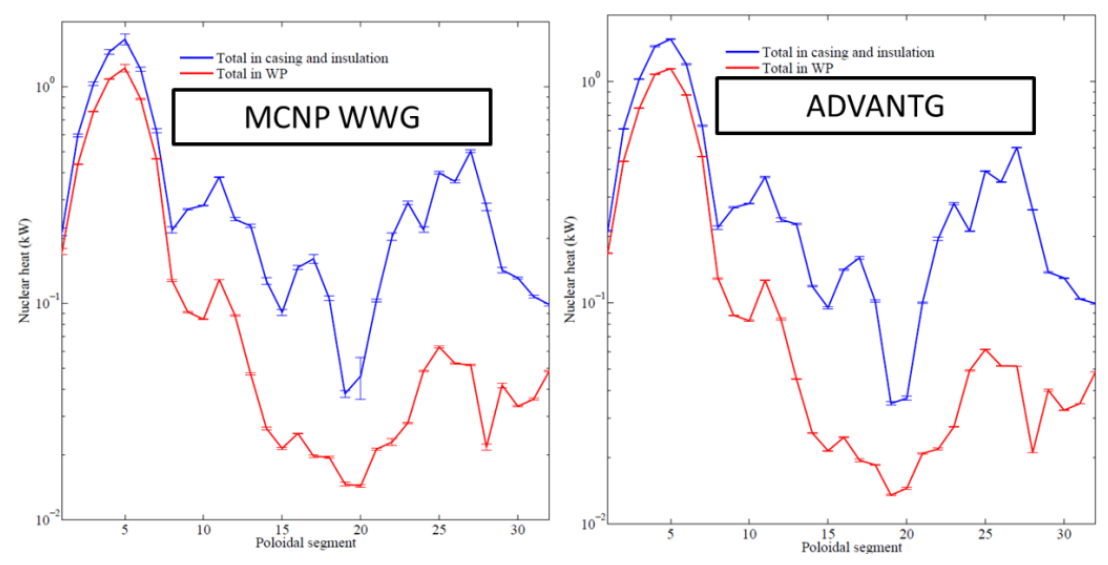

Fig. 2. Poloidal distribution of total nuclear heating in all coil casing and ground insulation components and the total nuclear heating in all the winding pack turns. The error bars represent $1 \sigma$ uncertainty.

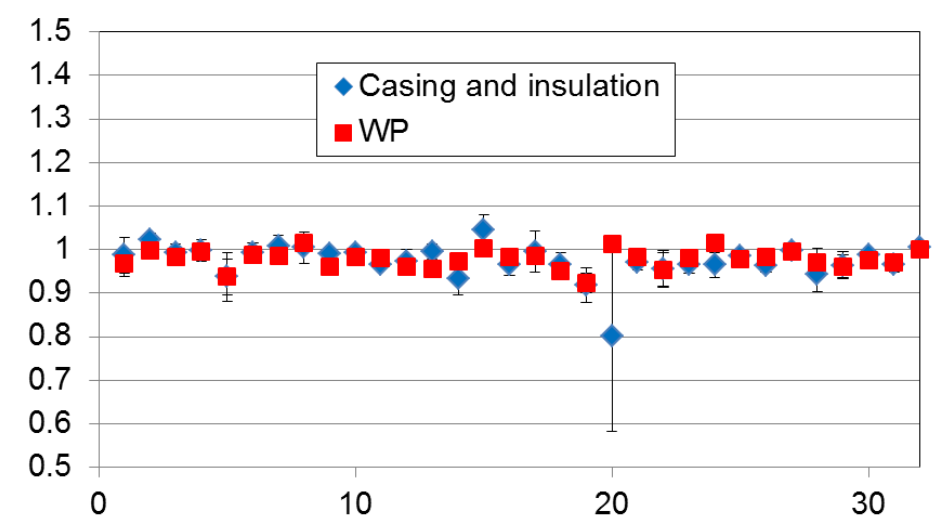

Fig. 3. Poloidal distribution of the ratio between the MCNP results that used the MCNP WWG to the MCNP results that used ADVANTG for total nuclear heating in all coil casing and ground insulation components and the total nuclear heating in all the winding pack turns. The error bars represent $1 \sigma$ uncertainty in the ratio. 

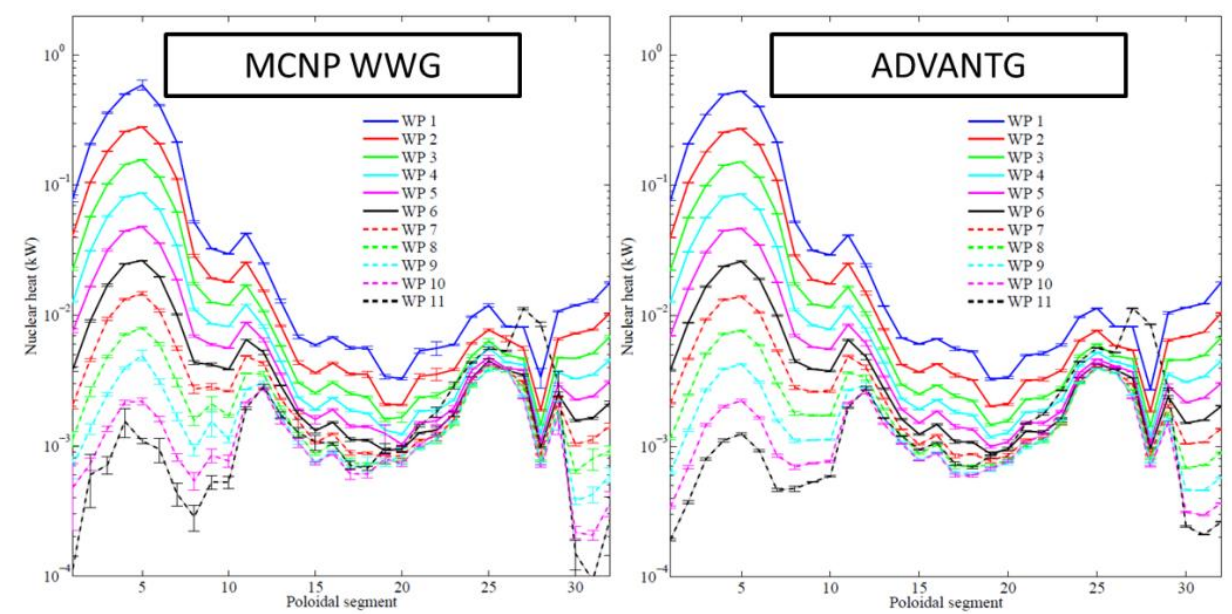

Fig. 4. Poloidal distribution of the nuclear heating in the individual turns of the TFC winding packs.

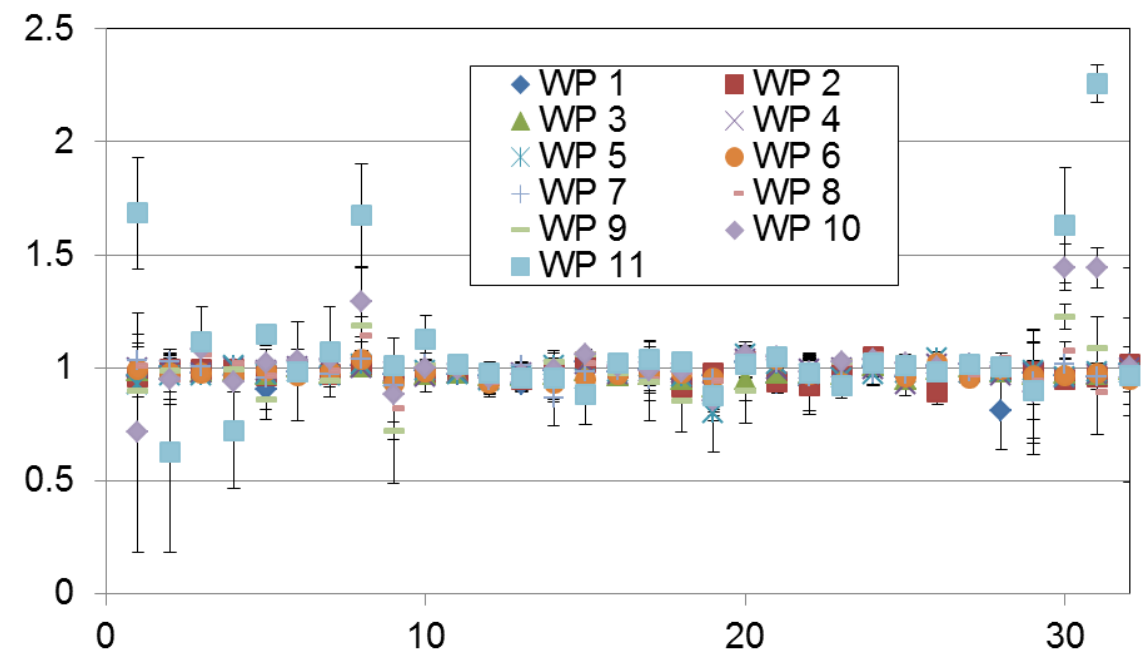

Fig. 5. Poloidal distribution of the ratio between the MCNP results that used the MCNP WWG to the MCNP results that used ADVANTG for the nuclear heating in the individual turns of the TFC winding packs. The error bars represent $1 \sigma$ uncertainty in the ratio.

For the total heating in all the winding pack turns, MCNP WWG and ADVANTG results did not agree within $3 \sigma$ for 7 segments out of the 32 . The relative statistical uncertainties of the MCNP WWG case varied between $0.2 \%$ and $4.1 \%$. The relative statistical uncertainties of the ADVANTG case varied between $0.2 \%$ and $1.3 \%$. The maximum difference between the MCNP WWG and ADVANTG results was $8.1 \%$, which was 5.1 times larger than the uncertainty in the average value. For all the segments, both MCNP WWG and ADVANTG results agreed within $3 \sigma$ with the analog results of which the relative statistical uncertainties varied between $0.7 \%$ and $13.2 \%$.

Figure 4 shows the poloidal distribution of the nuclear heating in the individual turns of the TFC winding packs and Fig. 5 shows the ratio of the nuclear heating calculated in these turns using MCNP WWG weight windows to the corresponding values calculated using ADVANTG weight windows. The relative statistical uncertainties of the MCNP WWG case varied between $8.5 \%$ and $53.0 \%$. The relative statistical uncertainties of the ADVANTG case varied between $0.3 \%$ and $4.7 \%$. The analog relative statistical uncertainties varied between $1 \%$ and $56.9 \%$. MCNP WWG and ADVANTG results did not agree within $3 \sigma$ in 9 out of the 352 tallies representing the 11 turns in the 32 segments. With the MCNP WWG, the nuclear heating in the $31^{\text {st }}$ poloidal segment of the $11^{\text {th }}$ winding pack turn was approximately a factor of 2.3 higher than the corresponding value calculated with ADVANTG. For this specific tally, the relative uncertainty of the MCNP WWG case was $8.1 \%$ and the relative uncertainty of the ADVANTG case was $1.0 \%$. These small relative uncertainties do not explain the large difference between these results because the difference in the results is approximately equal 14.9 times the uncertainty in the difference This shows that seemingly low relative uncertainty of either the MCNP WWG or the ADVANTG cases is clearly undersampled for this 
tally. However, it is clear from Fig 4 that the nuclear heating values calculated using ADVANTG do not suffer from the unphysical sudden variation at the $31^{\text {st }}$ segment of the $11^{\text {th }}$ winding pack while the MCNP WWG result do, which confirms that the undersampling occurred only in the MCNP WWG case. Additionally, the ADVANTG nuclear heating result for this tally was only $18.3 \%$ different from the corresponding result of the analog calculation while the MCNP WWG result was a factor of 2.8 higher than the analog result. The $18.3 \%$ difference between the nuclear heating tally of ADVANTG and the analog tally can be explained by the $54.0 \%$ relative uncertainty of this tally in the analog calculation, which suffered from high uncertainties in the $11^{\text {th }}$ winding pack turn because it is well shielded from the plasma source. Even though the high relative uncertainty makes the analog calculation of the nuclear heating in this winding pack turn unreliable, the agreement between ADVANTG and analog results in this tally segment increases the confidence in the ADVANTG results that did not suffer from the unphysical discontinuity that was apparent in the MCNP WWG case.

\section{Conclusion}

Because of the difficulty of MC calculations in tally segments with small volumes, the MCNP WWG was previously used by the ITER IO to speed up the MCNP calculation of the nuclear heating distribution in the different components of the coil casing, ground insulation, and winding packs of the ITER TFC. In this work, we utilized the ORNL ADVANTG code instead of the MCNP WWG to accelerate the MCNP calculation for quality assurance. Even though the ADVANTG MCNP calculation used less than one-sixth of the computational resources of the MCNP WWG calculation, the relative uncertainties of all the tallies were less than $6.1 \%$ with the ADVANTG calculation while the maximum relative uncertainty of the MCNP WWG calculation was $82.7 \%$. The difference between the MCNP WWG and ADVANTG results exceeded what is expected by the statistical nature of the MC method for some quantities calculated. This indicates that the statistical uncertainties associated with some of the tallies may be smaller than their true value for either of the MCNP WWG or the ADVANTG calculations, which indicates that either of the two calculations suffered from severe undersampling problems; however, the ADVANTG calculation is considered more reliable because it did not suffer from the unphysical sudden variations in the calculated mean value and statistical uncertainty. Moreover, the results of the ADVANTG MCNP case better agreed with the results of the analog calculation even though the analog calculation had very high statistical uncertainty in the back turns of the TFC winding packs that are well shielded from the plasma neutron source. For this reason, we recommend the use of ADVANTG for similar MCNP calculations of detailed distributions of nuclear responses.

\section{Acknowledgment}

This manuscript has been authored by UT-Battelle, LLC under Contract No. DE-AC05-00OR22725 with the U.S. Department of Energy. The United States Government retains and the publisher, by accepting the article for publication, acknowledges that the United States Government retains a non-exclusive, paid-up, irrevocable, world-wide license to publish or reproduce the published form of this manuscript, or allow others to do so, for United States Government purposes. The Department of Energy will provide public access to these results of federally sponsored research in accordance with the DOE Public Access Plan (http://energy.gov/downloads/doe-public-access-plan).

This work used an adaptation of the Clite MCNP model which was developed as a collaborative effort between the FDS team of the Chinese Academy of Science, the University of Wisconsin-Madison, ENEA FRASCATI, CCFE UK, JAEA NAKA, and the ITER Organization.

\section{References}

[1] ITER Magnet Design Description Document Part 1, ITER report, ITER_D_2NPLKM, version 1.8, 2009.

[2] E. Polunovskiy, Progress in TFC Analysis, ITER report, ITER_D_Q7NRZE, version 1.0, 2015.

[3] X-5 Monte Carlo Team, MCNP-A General N-Particle Transport Code, Version 5- Volume I: Overview and Theory, LA-UR-03-1987, Los Alamos National Laboratory, 2003.

[4] T. Booth and J. Hendricks, Importance Estimation in Forward Monte Carlo Calculations, Nuclear Technology, 5 (1984) 90-100.

[5] S. Mosher et al., ADVANTG-An Automated Variance Reduction Parameter Generator, Oak Ridge National Laboratory report, ORNL/TM-2103/416, 2013.

[6] T. Evans, A. Stafford, R. Slaybaugh, and K. Clarno, Denovo: A New Three-Dimensional Parallel Discrete Ordinates Code in SCALE, Nucl. Technol. 171 (2010) 171-200.

[7] J. Wagner and A. Haghighat, Automatic Variance Reduction of Monte Carlo Shielding Calculations Using the Discrete Ordinates Adjoint Function, Nucl. Sci. Eng. 128 (1998) 186-208.

[8] J. C. Wagner, D. E. Peplow, and S. W. Mosher, "FWCADIS Method for Global and Semi-Global Variance Reduction of Monte Carlo Radiation Transport Calculations," Nucl. Sci. Eng., 176 (2014) 37-57.

[9] A. Ibrahim, Automatic Mesh Adaptivity for Hybrid Monte Carlo/Deterministic Modeling of Difficult Shielding Problems, $\mathrm{PhD}$ thesis, University of Wisconsin-Madison, 2012.

[10] B. Kiedrowski and A. Ibrahim, Evaluating the Efficiency of Estimating Numerous Monte Carlo Tallies, Trans. Am. Nucl. Soc. 104 (2011) 325-329. 